\title{
Growth Cone "Collapse" in vivo: Are Inhibitory Interactions Mediated by Gap Junctions?
}

\author{
Laura R. Wolszon, Wei-Qiang Gao, ${ }^{a}$ M. Beatrice Passani, ${ }^{b}$ and Eduardo R. Macagno \\ Department of Biological Sciences, Columbia University, New York, New York 10027
}

In the leech embryo, oppositely directed axons of homologous anterior pagoda (AP) neurons overlap with each other extensively within the nerves that link adjacent ganglia, and inhibit each other's further growth (Gao and Macagno, 1987b). During this 5-8 d period of inhibition, the axons begin to grow thin, and eventually they retract completely. However, deletion of one overlapping AP cell results in the renewed growth of the remaining AP cell's axon, which then innervates territory vacated by the killed cell. Thus, each neuron can detect the presence of the other, and adjust its branching pattern accordingly.

To begin to explore how these neurons detect and inhibit each other, we tested for direct communication between them. Dye fills with fluorescent chromophores suggested direct contact between their axons at the light level, and this was confirmed by serial-section electron microscopic analysis. Morphological features resembling aspects of gap junctions were observed where the projections were closely apposed, and subsequent electrophysiological recordings demonstrated electrical coupling between the mutually inhibited axons. Confirmation that these projections communicate via gap junctions was obtained using intracellular injection of 5-HT as a tracer, followed by anti-5-HT immunohistochemistry. The tracer passed selectively between AP neurons.

We propose that the gap junctions formed between the transient projections of the developing AP neurons may mediate the exchange of the signals that permit homologs to recognize each other and to inhibit the further forward progress of these projections.

[Key words: CNS, development, retraction, electron microscopy, 5-HT, electrical coupling, leech, Hirudo medicinalis]

Interactions of growing neurons with surrounding cells are crucial to the proper development of the nervous system. These interactions are not always growth promoting, however. Indeed,

\footnotetext{
Received July 6, 1993; accepted Aug. 20, 1993

We thank Drs. Stan Kater, Ken Muller, and Vincent Rehder for helpful comments on the manuscript, and Nik Necles for assistance with the photography. This research was supported by NIH Grant NS20336 and NIH Grant BNS8819970 to E.R.M. and an NRSA postdoctoral fellowship to L.R.W.

Correspondence should be addressed to Laura R. Wolszon, Columbia University, Department of Biological Sciences, 1003 Sherman-Fairchild Center, New York, New York 10027.

a Present address: Department of Neuroscience, Genentech, Inc., South San Francisco, California 94080.

b Present address: Universitá degli Studi di Firenze, Dipartimento di Farmacologia Preclinica e Clinica, Viale Morgagni 65, 50134 Firenze, Italia.

Copyright (C) 1994 Society for Neuroscience $0270-6474 / 94 / 140999-12 \$ 05.00 / 0$
}

many recent experimental observations have revealed that inhibitory interactions are at least as important as growth-promoting ones in fine tuning the wiring of the nervous system (reviewed in Patterson, 1988; Schwab et al., 1993).

Most of the evidence for the existence of growth-inhibiting factors stems from in vitro observations of growth cone collapse or decreased neurite growth. Such factors include, on the one hand, soluble substances like the neurotransmitters 5-HT (Haydon et al., 1984) and dopamine (Lankford et al., 1988), and on the other hand, membrane-associated proteins found, for example, on myelin (Caroni and Schwab, 1988; Bandtlow et al., 1993) and cells of the CNS (Raper and Kapfhammer, 1990). The ways in which inhibitory signals are transduced are still unknown, although some evidence suggests that the regulation of calcium levels in growth cones may be important (Kater and Mills, 1991).

For the past several years, we have been exploring a specific inhibitory interaction among segmental homologs in the CNS of the medicinal leech, Hirudo medicinalis, whose large, identifiable neurons give us the opportunity to investigate developmental neuronal interactions in vivo. The neurons under study are the AP (anterior pagoda) cells, found as bilateral pairs in 20 of the 21 midbody ganglia (Fig. 1). Interestingly, all embryonic AP cells extend transient axons toward their homologs in adjacent ganglia, via the conneclive nerves. This growth pattern means that adjacent AP cells have axons that overlap with those of their neighbors from the 10th day of embryogenesis (E10) until E15-E1 8 (Gao and Macagno, 1987b). While overlapped, the AP axons inhibit each others' further growth, and then begin to retract. It is not as yet known what signal mediates either the initial inhibition or the subsequent retraction, but it is potent enough that generally by E25 the longitudinal axons disappear and the cell takes on its adult morphology (Fig. 1).

A striking finding is that if one AP cell is killed during the period of inhibition, the remaining axon resumes growth instead of retracting, and eventually takes over territory vacated by the dying cell (Gao and Macagno, 1987b; Gao, 1989). The signaling that occurs between these overlapping axons is therefore essential for the establishment of effective adult connections. It is for this reason that the elucidation of how transient AP axons communicate with each other, during the period of overlap, becomes extremely important.

We have used electrophysiology, electron microscopy, and dye coupling with 5-HT to show that the transient axons of adjacent AP cells communicate with each other directly, via gap junctions, during the few days that they are in contact. These findings then allow us to put forth the hypothesis that gap junctions might allow a signal to be exchanged between these axons during normal development, telling each neuron whether its 


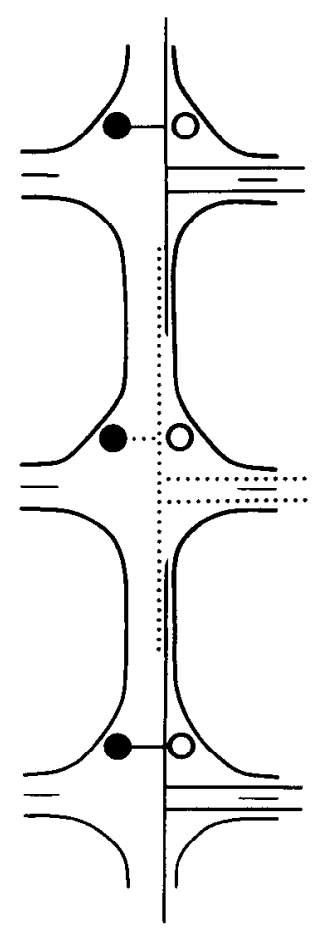

$\mathrm{E} 10-\mathrm{E} 14$

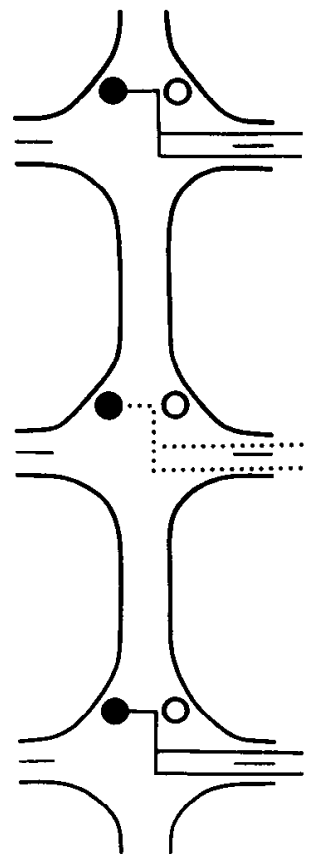

Adult

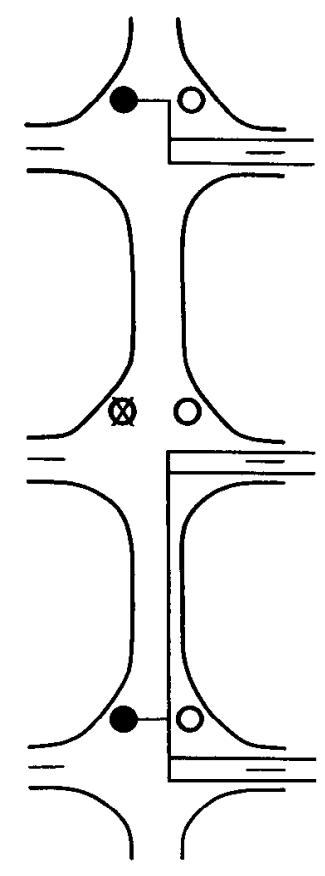

Kill $A P \leq E 14$

Figure 1. Schematic diagram of AP morphology in the embryo, adult, and an adult in which one AP was ablated before E14. The leech CNS is made up of head and tail ganglia and 21 midbody ganglia (MG), which communicate with each other primarily via a pair of connective nerves, running along the anteroposterior axis. Three adjacent midbody ganglia are illustrated here. The CNS is also connected to the periphery by two pairs of root nerves, one pair on each side of the ganglion. Each midbody ganglion is bilaterally symmetric and contains about $400 \mathrm{neurons}$, except for MG5 and MG6, which are specialized for sexual function (Macagno, 1980). Most of the neurons identified thus far, including the AP cells, are paired and have homologs in most ganglia. Left, Three adjacent AP neurons at E10-E14. Alternating cells are shown in solid and dotted lines; the mirror-image AP cells on the opposite side of the ganglion are illustrated only as somata (open circles), for simplicity. The AP5 cell's primary neurite crosses the midline within the ganglion, turns posterior, and branches, forming two lateral axons that exit via the contralateral roots, and longitudinal projections (that include two or more collateral axons) into each of the anterior and posterior contralateral connective nerves. The longitudinal projections from adjacent AP neurons overlap in the connective nerve and interact from E10 until E15-E18, when they begin to retract and finally disappear. The lateral projections remain into adulthood. Center, The adult morphology of three adjacent AP homologs. Note that the embryonic processes in the connective nerves have completely disappeared, though the lateral axons remain and innervate distributed territory in the body wall. Right the results of killing an AP cell in an intact embryo during E10-E14, the period that longitudinal axons overlap in the connective nerve. The photoablated cell is marked with an $X$. Most commonly, the posterior AP neuron reinitiates growth of the projection that faced the dying cell in the embryo (Gao and Macagno, 1987b), instead of retracting it. (The anterior AP responds less frequently, and occasionally, the two adjacent homologs share the vacated territory, each sending an axon out one of the lateral roots.) Note that longitudinal projections directed away from the killed cell were retracted as usual.

homolog is present and, therefore, how much territory to innervate.

\section{Materials and Methods}

Hirudo medicinalis embryos were raised in our laboratory as described in Gao and Macagno (1987a), except that the embryos were maintained at $23^{\circ} \mathrm{C}$. Embryogenesis is complete within $30 \mathrm{~d}$ at this temperature. Many neurons begin extending axons on or before E7 (W.-B. Gan, unpublished observations), but we worked with embryos from E10 onward because this was the earliest time at which we could consistently identify the AP cells, and at which the cells could withstand electrode penetration and dye injection.

Dissection of embryos. Embryos were first anesthetized in sterile, dilute Instant Ocean solution ( $0.5 \mathrm{gm} / \mathrm{liter}$; Menasha Corporation) at $2^{\circ} \mathrm{C}$ for $10 \mathrm{~min}$. Very long exposures to traditional invertebrate anesthetics such as ethanol or high magnesium sometimes adversely affected the electrical responsiveness of the neurons.

For embryos up to E13, the cryptolarval membrane, which envelops the cocoon fluid, was split along the longitudinal axis with fine forceps to remove the fluid. Older embryos and juveniles (E30-P1) had more viscous fluid, so these animals were pinned dorsal side up after opening, and the fluid removed using a stream of saline from a wash bottle. The preparation was then transferred to $6 \%$ ethanol in sterile Wenning's saline [40 mM DL-malic acid (Sigma; free acid form, neutralized to $\mathrm{pH}$ 7.0 with $\mathrm{NaOH}$ ), $4 \mathrm{~mm} \mathrm{KCl}, 10 \mathrm{~mm}$ disodium succinic acid (Sigma), $10 \mathrm{~mm}$ Tris base, $1.8 \mathrm{mM} \mathrm{CaCl}_{2}$, and $\mathrm{pH}$ adjusted to 7.4 with $\mathrm{NaOH}$; Wenning, 1987] on a microscope slide coated with Sylgard (Dow-Corning). The embryo was pinned to the Sylgard, ventral side up, using 001 tungsten pins (American Wire Industries). The elastic cryptolarval membrane of the embryo was stretched during pinning along the longitudinal and lateral axes in order to minimize movement of the germinal plate during recording; no conventional anesthetics that we tried stopped movement in these embryos. Contractions were further reduced by placing pins in both sides of the body wall, next to the ganglia from which the recordings were to be made, and then making short, lateral cuts in the body wall between segments on each side of the embryo. (Incidentally, this procedure also prevents peripheral interactions bctween AP cells in neighboring segments, if there are any.) If none of these treatments were sufficient to stop the contractions of the musculature, the embryo was immersed in $0.1 \%$ paraformaldehyde in sterile saline for $1 \mathrm{~min}$, and then immediately rinsed with saline. This procedure had no detectable effect on observed electrophysiological responses. A longitudinal incision was then made in the ventral body wall over 4-10 adjacent midbody ganglia using an etched tungsten pin. The remaining blood sinus was removed from the surface of the ganglia with the same pin.

Electrophysiological recording. We used an electrophysiology rig that 
had a microscope equipped with epifluorescence optics and a $40 \times$ waterimmersion lens. This magnification facilitated the identification of embryonic AP neurons, but necessitated bending the tips of the electrodes to a $45^{\circ}$ angle (using a heated nichrome wire) to accommodate the working distance of the objective. To verify the identity of the AP cells, we included Lucifer yellow dye (Sigma) in every electrode solution (3$8 \%$ in $0.2 \mathrm{M} \mathrm{LiCl}$, microfiltered) and used -1 to $-5 \mathrm{nA}$ currents (500 $\mathrm{msec}, 1 \mathrm{~Hz}, 2 \mathrm{~min}$ ) to fill the cells after termination of the experiment. These electrodes had resistances of 150-300 M , high enough to cause frequent blockage of the electrodes and often high levels of AC interference, but fine tips were necessary to minimize damage to the neurons.

Measurements of electrical coupling. To measure electrical coupling between AP cells, we recorded simultaneously from these neurons in adjacent ganglia while applying current pulses of 5-7 $\mathrm{nA}$ (positive and negative; $400 \mathrm{msec}$ duration). At the end of each experiment, to control for possible electrode cross talk or electrical coupling via extraneuronal pathways, we withdrew the electrode(s) from one or both cells and applied the same current pulses in the bath.

To reduce instability during recordings, the microscope stage was fixed in the z-axis. However, the $40 \times$ objective required for identifying young cells necessitated viewing one ganglion at a time; the stage therefore had to be movable in the other two dimensions. The resulting mechanical instability, coupled with the fragility of embryonic neurons, prevented our achieving long electrode penetrations; this often limited the number of current pulses we could apply in each cell pair to only a few. Reliable dual recordings from adjacent AP cells could therefore be obtained from E10-E12 embryos for no more than $1.5 \mathrm{~min}$ and from E13-E15 embryos for up to $3 \mathrm{~min}$, after which the resting potentials deteriorated rapidly and the electrical coupling diminished. Single-electrode recordings often lasted several minutes longer (up to $30 \mathrm{~min}$ for E16 embryos). Although data were digitized at $600 \mathrm{~Hz}$ for subsequent analysis, the restricted time available for stable recording prevented our being able to average repeated measurements digitally; therefore, our numbers were obtained from the first (and most reliable) traces of a series.

The 150-300 M $\Omega$ resistances of the electrodes caused frequent blockage of the tips, resulting in an instability of the electrodes that made bridge-balance difficult when using the rising phase of the presynaptic voltage change. To circumvent this problem, we instead bridge-balanced using the falling phase of the pulse, after ensuring that the pulse was long enough to allow the presynaptic cell to reach a nearly steady-state voltage. Since the time constant of decay of the electrode was significantly faster than that of the cell, there was usually minor difficulty determining the intersection of their two exponentials upon turning off the injected current. However, to be certain that visual examination of the falling phase accurately determined presynaptic voltage, we measured falling-phase voltage responses due to applied currents in adult AP cells, in which two high-resistance electrodes could be placed in a single cell. One (active) electrode was used both to pass current and to measure voltage with bridge balancing, and the other (passive) to measure voltage directly, without applying current. Comparison of the two measurements revealed that there was a root-mean-squared error of less than $5 \mathrm{mV}$ in response to negative current pulses when the electrodes were capacity compensated $(n=4$ experiments) and $15 \mathrm{mV}(n=3$ experiments) when they were not. For this reason, we made certain that the electrodes were well compensated before making measurements. Positive current pulses caused more blockage of the electrodes and produced unacceptably high levels of measurement error. As a further validation of this measurement technique, the falling-phase voltage responses were fit to two exponentials and the slow (cell) response extrapolated to time zero. These measurements of presynaptic voltages were nearly identical to those done by eye, in both embryos and adults, providing that we were working in the linear range of the current-voltage relationship. Outside of this range, negative pulses were more accurately measured by eye (as determined from three-electrode experiments in adults), whereas positive ones were more accurate using exponential fits. Finally, it was observed that for adult AP pairs with two electrodes in the presynaptic cell, measurements of coupling coefficients using the rising phase versus the falling phase of the voltage pulse were within $5 \%$ of each other.

Since the time available for intracellular recording in embryonic neurons was limited, we found it more convenient to bridge-balance digitally, after the recordings were stored on the computer, using the same criteria (e.g., determination of the intersection of two exponentials) as are traditionally applied during live recordings.

Intracellular dye injections. Embryonic neurons were filled with horse-

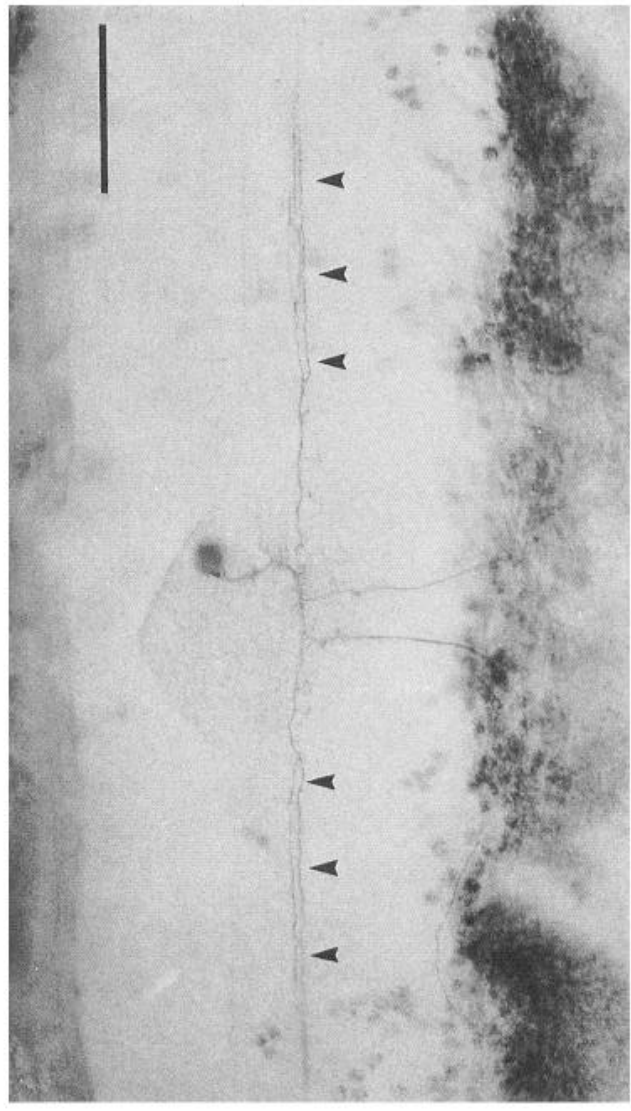

Figure 2. HRP-filled AP neuron in MG8 at E10. The longitudinal projections in the connective nerves branch into two or more axons (arrowheads) after they leave the margins of the ganglion. Scale bar, 100 $\mu \mathrm{m}$.

radish peroxidase (HRP; Sigma), rhodamine dextran amine (RDA; Molecular Probes; $2 \%$ in $0.2 \mathrm{M} \mathrm{KCl}$ ), or Lucifer yellow (3-8\%) by applying positive or negative current, as described above. The HRP and RDA were allowed to diffuse for at least $2 \mathrm{hr}$ before $30 \mathrm{~min}$ fixation with paraformaldehyde; the Lucifer yellow required only $15 \mathrm{~min}$. The preparations were then cleared in glycerol, mounted, and photographed with Kodak T-Max 400 or Ektachrome 400 film.

Injection and visualization of 5-HT. A solution of 5\% 5-HT (Sigma) in $0.2 \mathrm{M} \mathrm{KCl}$ and $0.05 \mathrm{M}$ Tris ( $\mathrm{pH} 7.4)$ was injected using $200 \mathrm{msec}$ pulses of positive current (1-2 nA, $2 \mathrm{~Hz}, 1-2 \mathrm{~min}$; Passani, 1992). The 5-HT was allowed to diffuse for $10 \mathrm{~min}$, after which the embryos were fixed for $1 \mathrm{hr}$ in $4 \%$ paraformaldehyde in $0.1 \mathrm{~m}$ phosphate buffer, $\mathrm{pH}$ 7.4. They were subsequently incubated overnight at $4^{\circ} \mathrm{C}$ in anti-5-HT monoclonal antibodies (provided by Drs. Kathy French and William Kristan, University of California at San Diego) that were diluted 1:1000 with $0.01 \mathrm{M}$ PBS and $1 \%$ Triton X-100. After rinsing with PBS, the antibodies were visualized using the ABC Vector kit (Vector Laboratories). This procedure involved incubating the tissue in biotinylated secondary antibodies (diluted 1:200 in 0.01 M PBS with $1 \%$ Triton-X) for $2 \mathrm{hr}$, rinsing, and then incubating in Texas red-conjugated avidin (3:100 in 0.05 м HEPES buffer, $\mathrm{pH} 8.2$ ) for another $2 \mathrm{hr}$. Finally, the preparation was cleared in a glycerol series, mounted, and photographed. Under these conditions, we were unable to achieve 5-HT immunoreactivity in adults or in embryos older than E20.

Photooxidation of Lucifer yellow and electron microscopy. Axons were stained for examination at the ultrastructural level by photooxidizing diaminobenzidine (DAB; Maranto, 1982). Two AP neurons in adjacent ganglia were filled with Lucifer yellow and then fixed in $4 \%$ paraformaldehyde in $0.1 \mathrm{M}$ phosphate buffer $(\mathrm{pH} \mathrm{7.4)}$ for $30 \mathrm{~min}$. The preparation was immersed in saturated, filtered DAB in $0.1 \mathrm{M}$ Tris buffer (pH 8.2), and moved to a Leitz microscope equipped with a $75 \mathrm{~W}$ arc lamp and a $20 \times$ objective. It was there illuminated for $40 \mathrm{~min}$ with $\sim 490 \mathrm{~nm}$ light to photoconvert the dye. During the exposure, photographs were taken in order to localize regions of interest, to be sectioned 

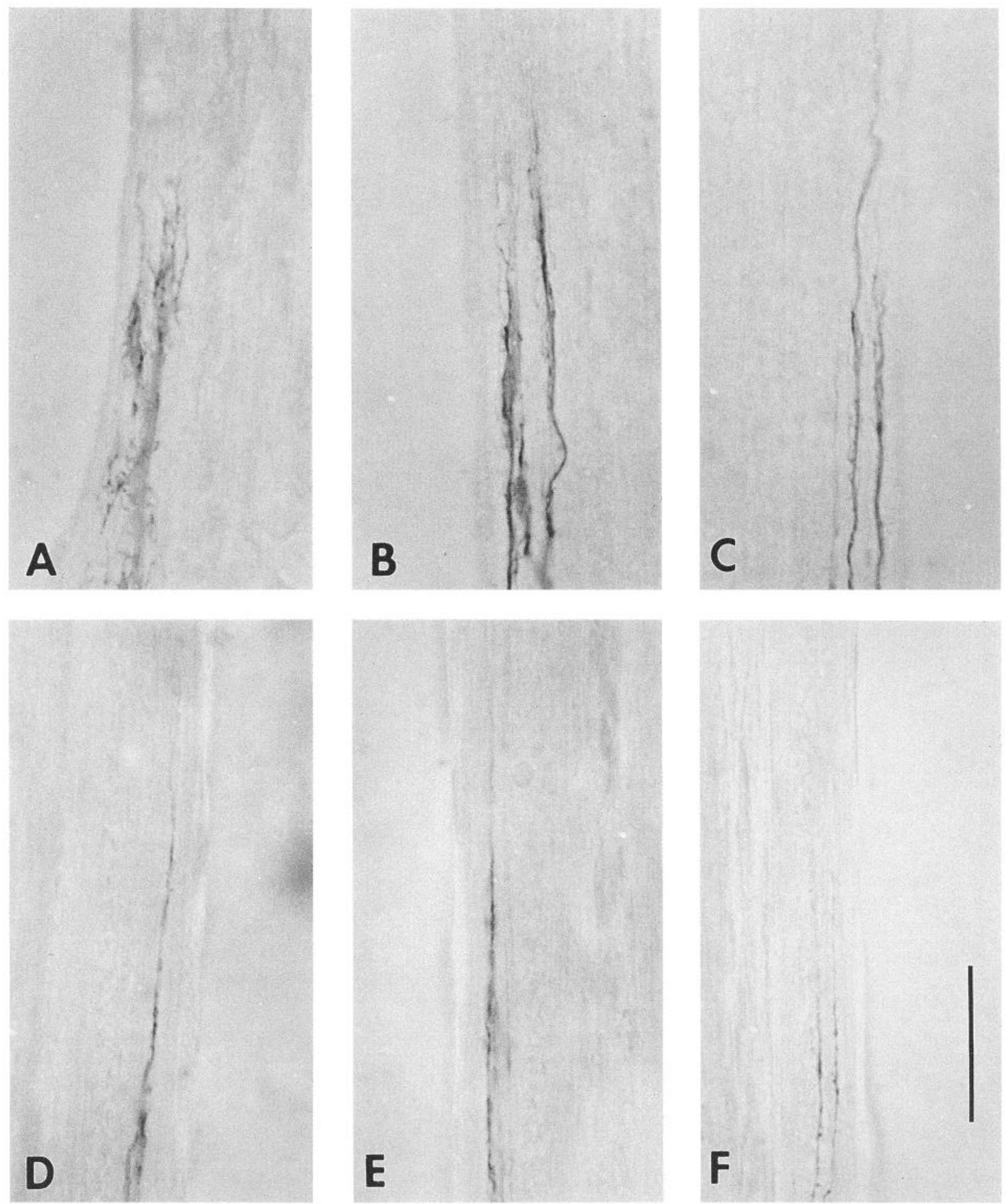

Figure 3. High-magnification photomicrographs of the growth cones of AP cells' longitudinal projections, filled with HRP. $A-F$ represent the growing tips from AP cells at E9, E10, E11, E12, E13, and E14, respectively. The morphology of growth cones in $A$ and $B$ resembles that expected of an elongating axon, while at later times the growth cones begin to lose their filopodia $(C$ and $D)$, get thinner $(D$ and $E)$, and retract $(F)$. Scale bar, $20 \mu \mathrm{m}$.

serially. The tissue was then postfixed for $2 \mathrm{hr}$ on ice in a freshly made solution containing equal amounts of $5 \%$ glutaraldehyde and $1 \%$ osmic acid in $0.1 \mathrm{M} \mathrm{Na-cacodylate} \mathrm{buffer} \mathrm{(pH} \mathrm{7.4).} \mathrm{After} \mathrm{rinsing} \mathrm{in} \mathrm{the} \mathrm{same}$ buffer, the preparation was prestained in ice-cold $0.5 \%$ uranyl acetate in $0.1 \mathrm{~m} \mathrm{Na}$-maleate buffer ( $\mathrm{pH} \mathrm{6.0)}$ ) for $90 \mathrm{~min}$, and was then washed, dehydrated, and embedded in Epon. Sections 80-90 nm thick were cut on an ultramicrotome (Sorvall MT 5000) and collected in ribbons of five or six sections each on Formvar-coated slot grids (Stevens and Trogadis, 1984). After poststaining with lead citrate $(20 \mathrm{~min}$ at room temperature), the sections were examined and photographed with a JEOL 1200EX electron microscope.

Computer reconstruction of electron microscope images. The serial electron micrographs were aligned with each other and rephotographed onto a $35 \mathrm{~mm}$ filmstrip, from which reconstructions of the labeled 


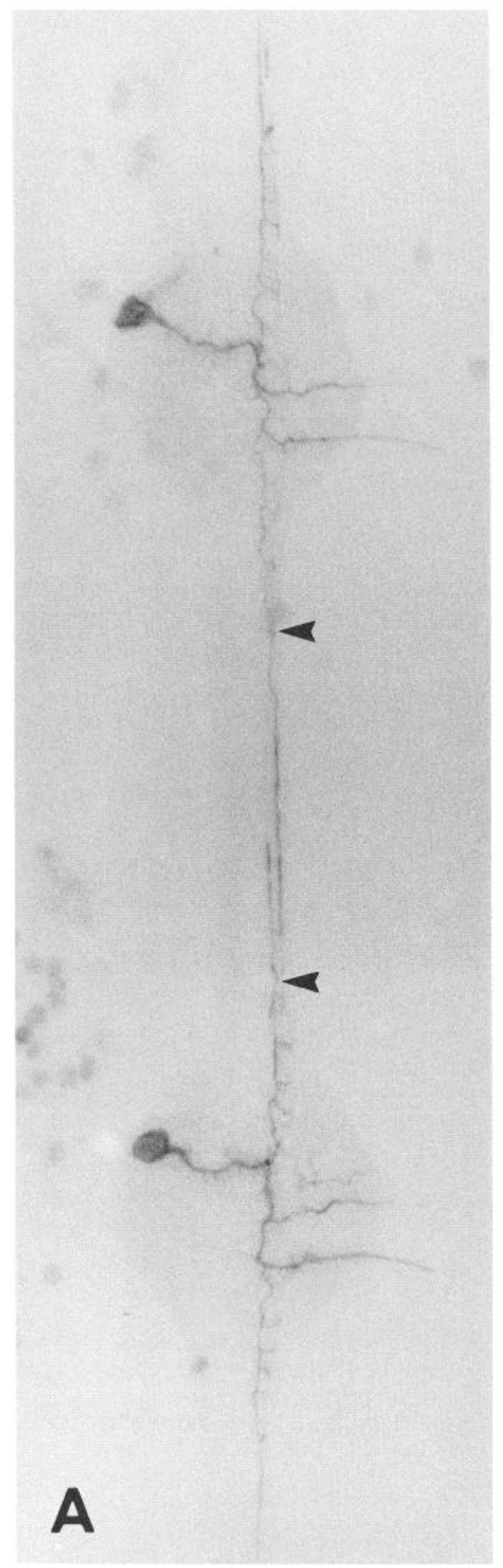

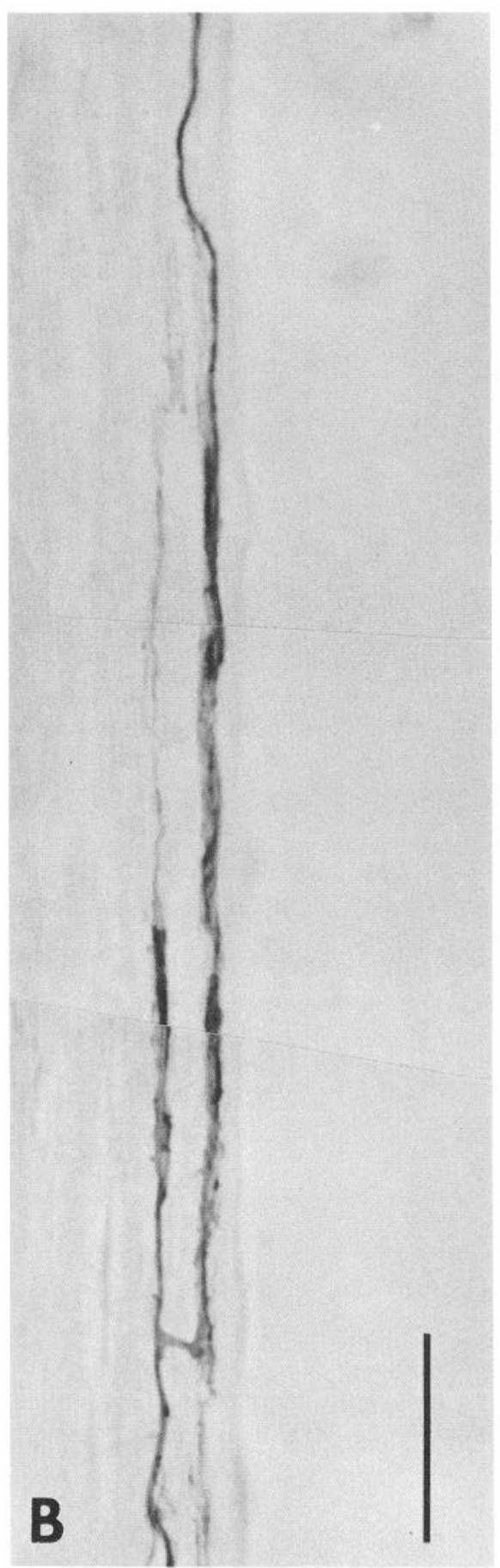

Figure 4. Transient projections of adjacent AP cells overlap with each other in the connective nerve. $A$, Two AP cells at E11, filled with HRP in a wholemount preparation to reveal the embryonic morphology. There are numerous small side branches of the longitudinal axons, formed near the exits of the ganglia, and sometimes in the connective nerves, where they often link the axons from both cells. The oppositely directed axons follow parallel paths within the contralateral connective nerve, their overlap covering roughly two-thirds of its span. The arrowheads demarcate the upper and lower limits of the region shown at higher power in $B . B$, High-magnification view of overlap between oppositely directed AP axons in the connective nerve. Note that, at this focal plane, a side branch of one of the axons appears to contact its neighbor. What is also common is that opposing axons intertwine with each other, not obvious here because of both cells being filled with the same dye (compare Fig. $5 A$ ). Scale bar: $1000 \mu \mathrm{m}$ for $A, 20 \mu \mathrm{m}$ for $B$. processes were produced using the CARTOS system (Macagno et al., 1979). These were displayed using an Evans and Sutherland PS 300 graphics station and photographed on Kodak Ektachrome film.

\section{Results}

Initial morphology of transient AP axons. Each longitudinal projection of an AP cell began as a single fiber and remained so while it was still confined to the region of the ganglion. However, the axon usually bifurcated after it left the margin of the ganglion, forming two or more branches of unequal lengths that grew along parallel paths past the middle of the contralateral connective nerve (Fig. 2), where they halted as though inhibited from growing farther. (For convenience, we sometimes refer to an AP cell's processes within one connective nerve as being a single "projection," but the reader should keep in mind that there are two or more axons in each projection.) Anterior axons were almost always longer than posterior ones, which may be significant in light of the anterior-to-posterior direction of germinal plate development.

At high power, each axonal branch had many small side branches, and terminated in a single growth cone crowned by numerous filopodia. The appearance of these growth cones during process outgrowth is discussed in the next section.

Changes in growth cone morphology upon encounter with ho- 

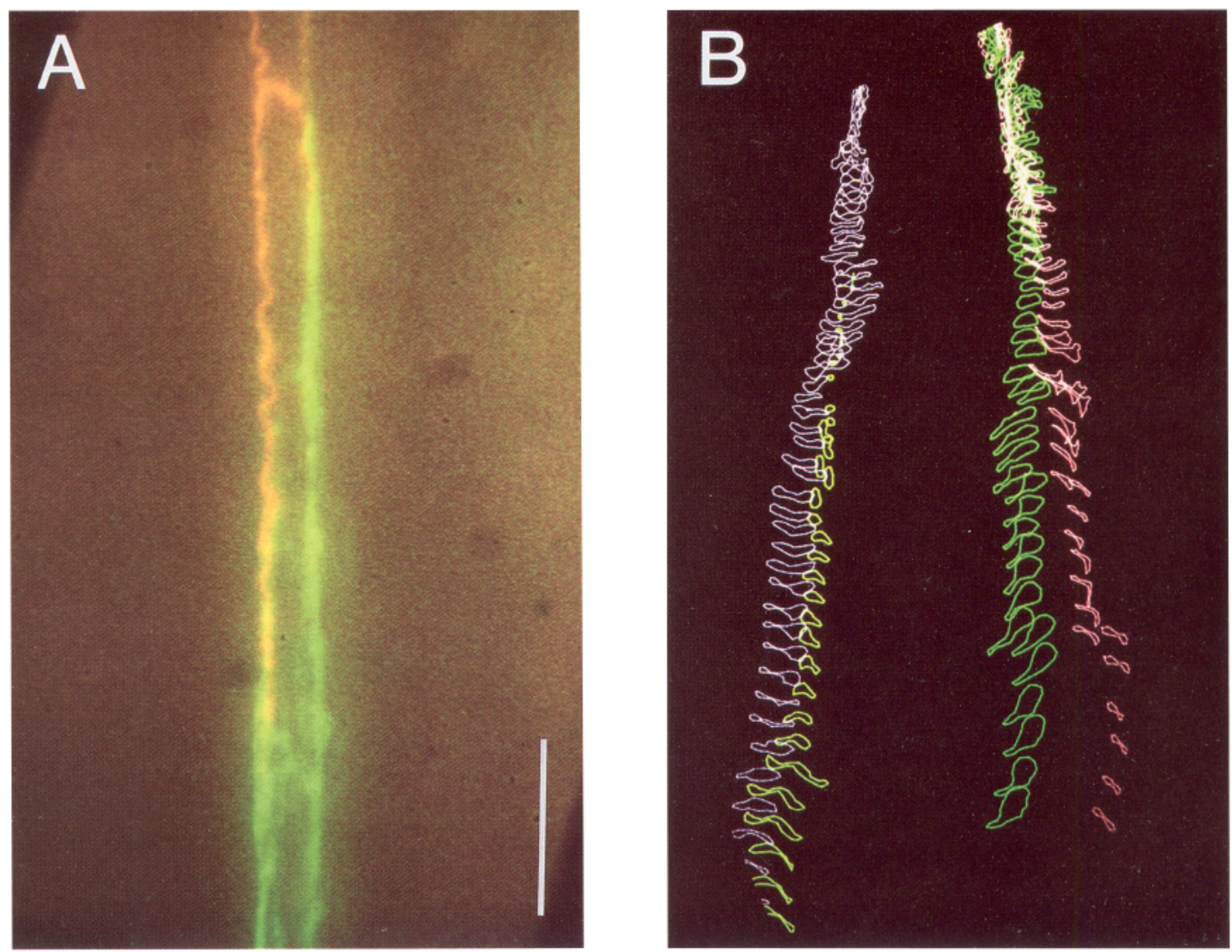

Figure 5. A, High-power view of overlapping axons from two adjacent AP neurons, the posterior one filled with Lucifer yellow and the anterior one filled with RDA. Only a region in the connective nerve is shown. In this preparation, each longitudinal projection is composed of two axons of different lengths. The regions in which oppositely directed axons overlap are orange, yellow, or light green (as opposed to pure red or pure green). $B$. Computer display of the processes of two AP homologs in adjacent ganglia (two axons from each projection), reconstructed from aligned serial electron micrographs as described in Materials and Methods. The branches from the posterior cell are shown in greens, those from the anterior cell in purples. Regions of close membrane apposition are quite extensive and appear white in places where the colors overlap. The left branch of the posterior cell (lighter green) terminates in the region shown here, but the other three branches displayed travel through it. The region shown corresponds approximately to the central fifth of the region of overlap shown in $A$. The serial sections from which the neurite perimeters were drawn, however, spanned the full length of the connective nerve between the ganglia, allowing the assignment of neurites to each of the two marked neurons. Scale bar: $150 \mu \mathrm{m}$ for $A, 35 \mu \mathrm{m}$ for $B$.

molog. When the growing tips of either longitudinal or lateral axonal projections of AP neurons were examined at E9, they displayed features typical of active growth cones, including flattened regions (lamellipodia) and numerous filopodia (Fig. 3A). The morphology of the growth cones of the longitudinal projections, however, began to change within a day or two, as they grew farther into the connective nerves and encountered the oppositely directed projections of the adjacent AP homologs. As shown by the examples from E10 to E14 embryos presented in Figure $3 B-F$, there was a dramatic loss of filopodia and lamellipodia with time, as the tips got thinner and eventually appeared to atrophy and regress. While the growth cones seemed to become largely inactive and to stop advancing during this period, it should be noted that at the same time the distance between the ganglia increased severalfold, so the stopped axons were actually becoming longer. Therefore, membrane was presumably being inserted along the length of the axons (Popov et al., 1993).

Ultrastructure of contacts between adjacent AP axons. When two adjacent AP homologs were filled with HRP (Fig. 4) or with two different fluorescent dyes (Fig. 5A), there were many apparent points of contact between the oppositely directed projections within the connective nerve. To verify that these axons were indeed in direct apposition, and to examine the nature of those contacts, we used photoconversion of fluorescent dye to produce an electron-dense reaction product in E1 1 embryos (see

Figure 6. Electron micrographs from a serially sectioned connective nerve containing the labeled processes of two adjacent AP homologs in E11 preparations. The projection from each neuron contains two axons. The cells were initially filled with Lucifer yellow, and the dye then photoconverted to yield an electron-dense reaction product as described in Materials and Methods. A, Low-power micrograph showing a cross section in the middle of the nerve. Each arrow indicates a pair of axons, one from each cell, traveling in parallel fascicles through this cross section. This section corresponds to about the middle of the reconstruction shown in Figure $5 B$. $B$, Higher-magnification electron micrograph showing the region surrounding the lower arrow in $A$, in an adjacent section. The processes of the two cells are labeled 1 and 2 . Both processes are flattened at this level (lamellipodia?) and show extensive areas of contact with one another. A glial cell profile is indicated with a $G$. Cl and $C 2$, High-magnification 

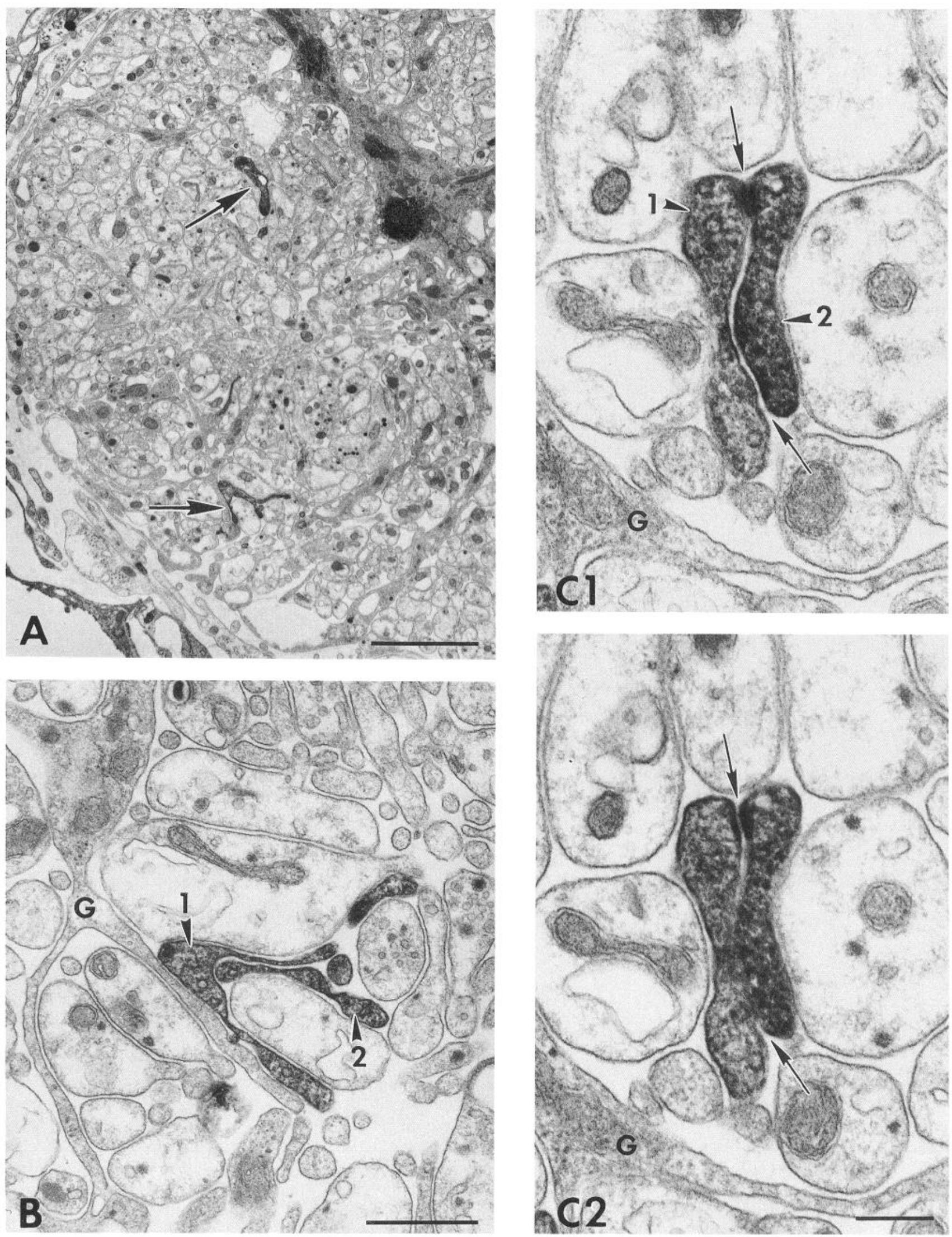

electron micrographs from a different preparation than shown in $A$ and $B$, showing close membrane apposition (arrows) between the processes of two AP neurons, labeled $I$ and 2 . A membrane thickening corresponding to a putative junction of unknown character can be seen at the top of the contact region in $C 2$. The panels show two tilt angles, about $25^{\circ}$ different from each other, of the same section. A glial cell profile is indicated by a $G$. Scale bars: $A, 2.5 \mu \mathrm{m} ; B, 0.5 \mu \mathrm{m} ; C 1$ and $C 2,0.2 \mu \mathrm{m}$. 

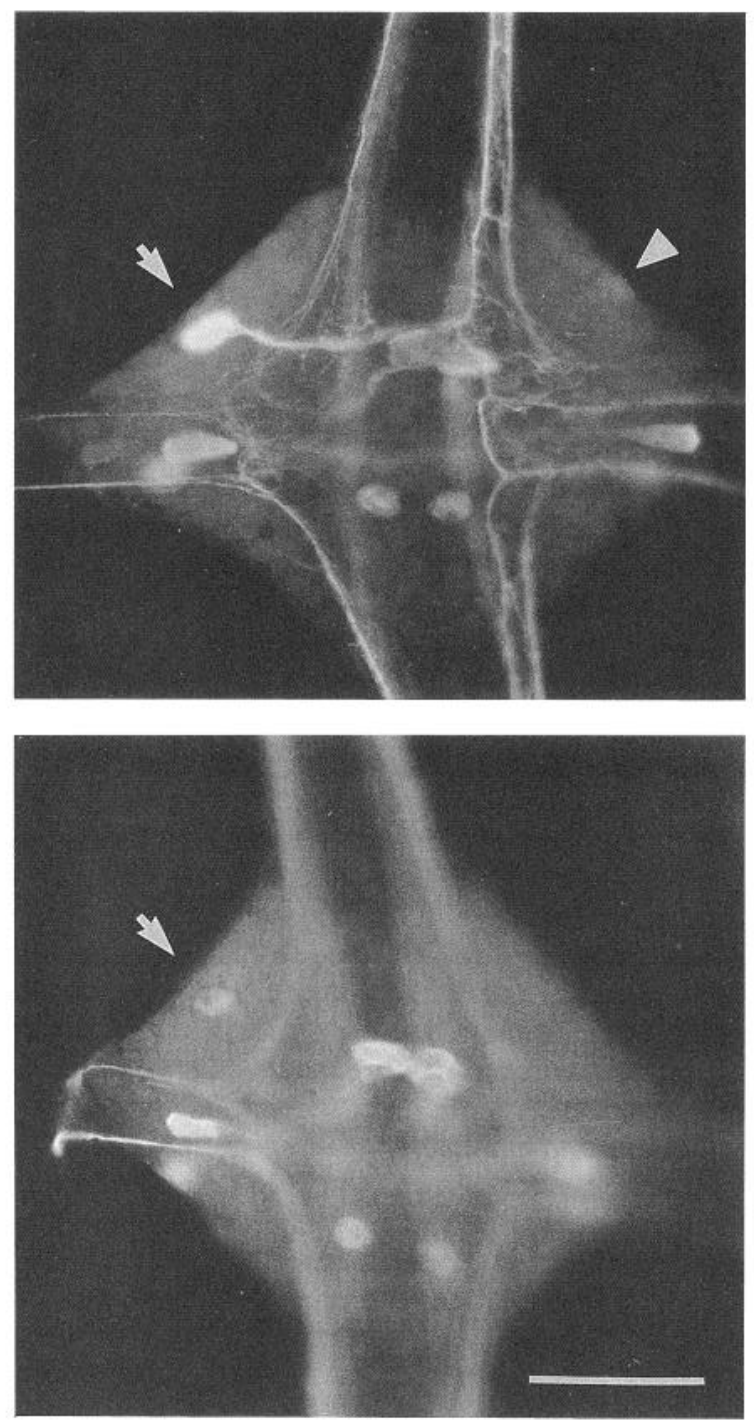

Figure 7. Developing AP neurons are dye coupled with 5-HT. Top. Photomicrograph shows the result of 5-HT injection into the left AP cell (arrow) at E11, and subsequent visualization of 5-HT with anti-5HT monoclonal antibodies. All of the other labeled neurons contain endogenous 5-HT, though the AP has none. The contralateral AP cell (arrowhead) shows barely discernible 5-HT staining (appears faint because the cell is in a different focal plane) resulting from the crossing of the dye via gap junctions between contralateral AP homologs (L. R. Wolszon, M. B. Passani, and E. R. Macagno, unpublished observations). Bottom, In the same embryo, but one ganglion posterior, 5-HT that diffused from the injected cell (top panel) into the longitudinal axons in the connective nerve, crossed into the posterior homolog on the ipsilateral side (arrow). Since contact between these homologs was prevented anywhere but in the connective nerve, the dye must have crossed from the anterior AP to the posterior AP via the gap junctional contacts between them. Scale bar, $50 \mu \mathrm{m}$.

Materials and Methods), and studied the ultrastructure of these axons in the connective nerve. This approach proved to be quite useful because the nearly inevitable difference in the degree of dye filling for the two neurons resulted in a darker reaction product in one of the two projections, simplifying the identification of profiles in electron micrographs. However, we confirmed the axonal identity in several experiments by making serial sections of the overlap region and following axonal profiles from section to section. For a few of these series, the CARTOS

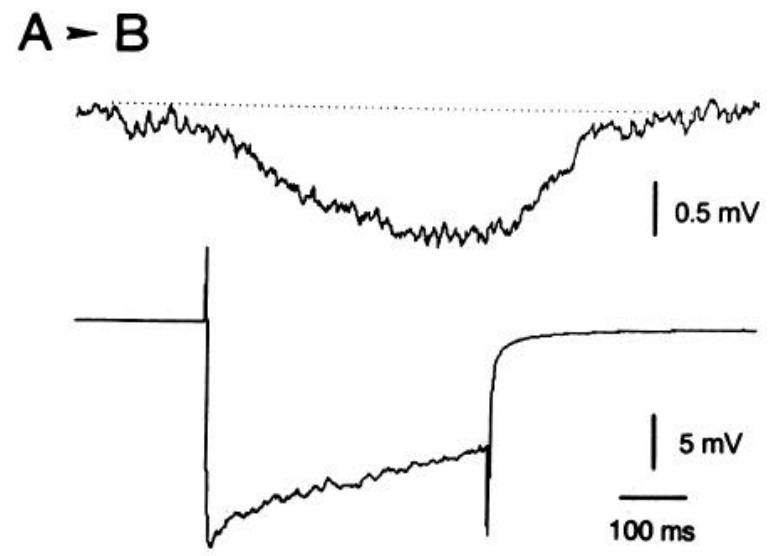

\section{$B=A$}

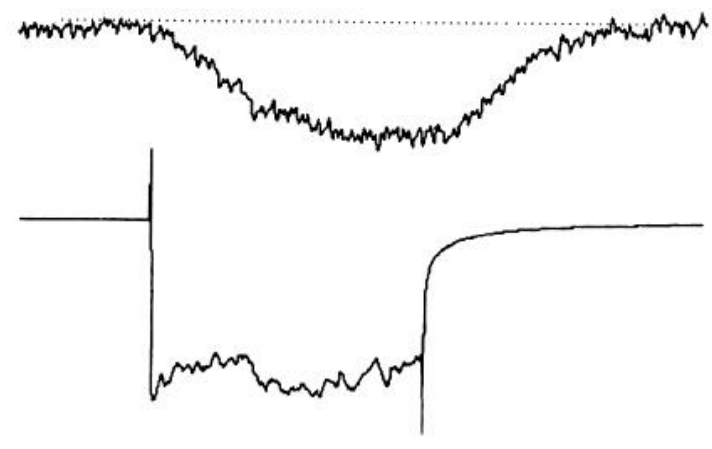

i

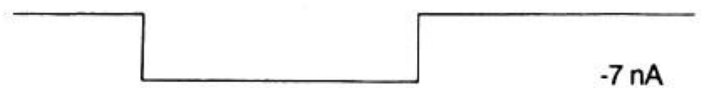

Figure 8. Simultaneous intracellular recordings from two AP cells in adjacent ganglia in an E11 embryo. A $-7 \mathrm{nA}$ current pulse was applied first to one cell $(A \triangleright B)$ and then the other $(B \triangleright A)$ while measuring the resultant $\delta V$ produced in each cell. The low-gain traces are from the injected cells, and are not bridge-balanced during the recordings. The noise in the traces is due to the very high resistances of the electrodes; this necessitated measuring coupling coefficients using the falling phases of the voltage responses (see Materials and Methods). Voltage and time calibration applies to both recordings. Dotted lines indicate the baselines.

system (Macagno et al., 1979) was used to reconstruct the region of overlap (see Materials and Methods). A part of one such reconstruction is shown in Figure $5 B$. In this preparation, each longitudinal projection, the one going anterior and the one going posterior, consisted of two parallel axons, for a total of four neurites. Only the largest branches were drawn, and hence no filopodia are visible. It can be seen in this figure that for each pair, an axon met its homolog, contacted it directly for part of its length, and then moved away again. There were several regions of contact between each pair of neurites, where the direct exchange of inhibitory signals might take place.

A low-power electron micrograph of a cross section of the connective, from the preparation illustrated in Figure $5 B$, is shown in Figure $6 \mathrm{~A}$. Each arrow indicates a site where a posterior-going axon contacted an anterior-going one; there are therefore four axons highlighted by these two arrows. The lower 
pair of axons, from an adjacent section, are shown at higher magnification in Figure $6 B$. The axons from the anterior cell (1) and posterior cell (2) can be clearly seen to be directly apposed over a significant fraction of their perimeters. The contact region also extends over a significant length of the two processes, as was seen in the computer reconstruction presented in Figure $5 B$.

Another example of the close relationship between the processes of the two homologs is shown in Figure 6, $C 1$ and $C 2$. Here, two tilt angles are presented in order to show the closeness of the apposed membranes and the existence of membrane specializations (arrows) in the contact regions. Since the DAB reaction product obscures the ultrastructure to a certain extent, the details of these specializations and of submembranous regions are not discernible. However, we did not expect to find structures between these axons resembling vertebrate gap junctions, as these have not been found as yet in the leech except between glial processes and between muscle cells (Coggeshall, 1974; Pumplin and Muller, 1983).

5-HT crosses gap junctions between embryonic AP cells. Most adult leech neurons that are electrically coupled are not dye coupled when Lucifer yellow or 6-carboxyfluorescein is injected into cells (reviewed in Muller et al., 1981). In an attempt to determine whether electrically coupled embryonic AP neurons are also dye coupled, we injected a series of small tracer molecules into these cells from E9 to E14. These tracers included Lucifer yellow, 6-carboxyfluorescein, biocytin, neurobiotin, K-dicyanoargentate, fura-2 (pentapotassium salt), and finally, 5 -HT $(\mathrm{MW}=212 \mathrm{Da})$. Of these, only 5-HT was found to cross between cells, as visualized with anti-5-HT antibodies, and then only weakly and infrequently (Passani, 1992). The best example is shown in Figure 7. Usually, the dye passed easily into the contralateral AP, within the same ganglion (arrowhead, top), but occasionally we observed it to traverse the length of the connective nerves (about $200 \mu \mathrm{m}$ ), cross between cells, and diffuse into the soma of the adjacent homolog (arrow, bottom). These findings, together with experiments showing that larger molecules do not pass between these cells, confirm that the transient projections connecting adjacent AP neurons are linked by gap junctions, and illustrate the usefulness of the 5 -HT/anti5-HT technique for detecting gap junctions between cells that are not dye coupled with conventional tracers.

Adjacent AP cells are electrically coupled. To test further the idea that the transient axons of AP cells are connected by gap junctions, we looked for electrical coupling between them by recording electrophysiologically from adjacent AP cells in vivo. We considered the cells coupled when there was a postsynaptic voltage change that paralleled the polarity and time course of the presynaptic one (Fig. 8) and that was significantly larger than (1) the electrode noise and (2) voltages produced by control current pulses in the extracellular space (see Materials and Methods). Since the somatic recording site was electrotonically distant from the synaptic sites in the neuropil, eliminating the possibility of voltage clamping these neurons in vivo, we did not attempt rigorous quantitation of the electrical communication. Nevertheless, we found that the majority of adjacent AP cell pairs were electrically coupled at ages when they still had overlapping axons in the connective nerve (through E15; Table 1). There was no coupling in older AP cells (E30-P1) whose axons had already retracted. This finding, in conjunction with the fact that the dissection technique prevented peripheral interactions between cells (see Materials and Methods), confirmed that elec-
Table 1. Number of AP pairs electrically coupled

\begin{tabular}{lcc} 
Age & \# Tested & \# Coupled \\
\hline E10 & 4 & 1 \\
F.11 & 8 & 7 \\
E12 & 3 & 1 \\
E13-E15 & 2 & 2 \\
Total embryonic & 17 & 11 \\
E30-P1 & 7 & 0 \\
(Postembryonic) & &
\end{tabular}

(Postembryonic)

trical coupling occurred within the CNS. The transient axons therefore communicate with each other directly, via gap junctions, during the few days that they are in contact.

We chose five of the best experiments, in terms of resting membrane potential and electrode performance, in order to estimate the degree of electrical coupling between neurons at E1 1. The coupling coefficient, defined as the postsynaptic voltage change (due to an applied current in the presynaptic cell) divided by the presynaptic voltage change, ranged from 0.007 to 0.069 and averaged $0.027 \pm 0.025( \pm \mathrm{SD})$ for these five experiments (see Materials and Methods for a description of how presynaptic voltage changes were measured). Since the recording sites were distal to the electrical synapses, these numbers almost certainly underestimate the degree of electrical coupling between adjacent AP neurons. Input resistances averaged $6 \mathrm{M} \Omega$ for $\mathrm{E} 11$ cells and $38 \mathrm{M} \Omega$ for E30-P1 cells.

\section{Discussion}

We report here that transient axonal projections, formed in connective nerves between adjacent AP neurons, communicate directly with each other early in development. Their growth cones, initially displaying a large number of filopodia extending in many directions, were found to thin and retract after making physical contact with their neighbors. We further report that the temporary axons communicate with each other via gap junctions during the period that they overlap. Ultrastructural studies revealed many sites of direct apposition between axons, and 5-HT, a small molecule that was used in these studies as a dye, diffused across the junctions. Finally, electrophysiological recordings indicated that adjacent AP neurons were electrically coupled during the period of overlap, but became uncoupled after the axons had retracted.

The changes in morphology of the growth cones after they encountered the oppositely directed neurites of the homologous neuron are both interesting and suggestive, particularly because they were observed in vivo. Numerous filopodia and flattened extensions or lamellipodia are hallmarks of actively extending growth cones both in vitro and in vivo (reviewed in Letourneau et al., 1992). Thus, their disappearance in the AP neuron indicates a change in the growth rate of the growing tip, and is consistent with the observation that the forward progress of the neurite toward the adjacent ganglion is severely curtailed or stopped altogether. The loss of filopodia and lamellipodia is also reminiscent of the changes observed in vitro when growth cones contact neurites of a different cell type (e.g., Kapfhammer and Raper, 1987a,b) or are exposed to purified membranes that carry an inhibitory factor (e.g., Raper and Kapfhammer, 1990). Two important differences are worth noting, however. First, the time scale of the growth cone collapse is very different in the animal 
and in the dish (days vs minutes), and second, the effects are produced in the leech by an interaction between processes of homologous neurons, while in general the work with vertebrate cells in vitro reveals growth-inhibiting effects only when the interactions are between heteronymous elements. Nonetheless, the similarities in the morphological changes suggest that the inhibitory signals, which are probably themselves quite different in the two cases under discussion, produce similar effects on the integrity of the cytoskeleton, leading to its depolymerization or collapse.

Gap junction morphology. Leech gap junctions thus far have only been identified unequivocally using freeze-fracture techniques (Pumplin and Muller, 1983). In that study, gap junctions resembling those in vertebrates were found between muscle cells and between successive wrappings of single glial cells. Although it is desirable to demonstrate the presence of gap junctions between neurons using freeze-fracture, it would be very difficult, if not impossible, to obtain the correct plane of fracture for two overlapping embryonic AP axons, within the connective nerve. Nonetheless, our anatomical studies do demonstrate that the AP processes have extensive contact regions, and that the membrane specializations and the sizes of the gaps between cells are consistent with there being gap junctions in these areas.

Electrical coupling without dye coupling. It is not surprising that of all dyes tested, only 5-HT passed between adjacent AP neurons, and then only infrequently. Not only are the gap junctional pores likely to be very small (see below), preventing access to the coupled cell, but the 5-HT was probably completely metabolized in many of the cell pairs long before it could diffuse the great distance to the soma of the adjacent homolog.

By far, the majority of leech neurons that are electrically coupled are not dye coupled with conventional dyes (Muller et al., 1981). Even neurons with extremely high coupling coefficients, such as the adult Retzius neurons, whose coupling coefficient ranges from $0.5-0.8$ in situ to nearly 1.0 in vitro (Ready and Nicholls, 1979), do not pass Lucifer yellow or carboxyfluorescein between them. There are some notable exceptions, however. Cell 151, also known as the "groundwire cell," is electrically coupled to almost every identified motor neuron in the ganglion (Wadepuhl, 1989) and, while dye cannot pass from Cell 151 to the motor neurons, Lucifer yellow or hexamine cobaltic chloride passes freely between groundwire homologs within one ganglon and to the homologs in adjacent ganglia. Similarly, S cells freely exchange Lucifer yellow with one another (Scott and Muller, 1980) and with a pair of interneurons (Muller and Scott, 1981; McGlade-McCulloh and Muller, 1989), although not with the $T$ sensory neurons, to which the latter are also electrically coupled. This variation in the degree of dye coupling raises intriguing questions about whether there are different types of gap junction proteins in different cells (or within a single cell), and whether those differences are manifested in divergent functions.

Electrical coupling in the absence of dye coupling has been observed in a number of other systems, both vertebrate and invertebrate. Audesirk et al. (1982) found that well-coupled neurosecretory neurons in the snail Lymnaea stagnalis rarely pass detectable amounts of Lucifer yellow, yet the ability to pass dye is not correlated with either coupling coefficient or input resistance. Studies in the marine mollusk Aplysia californica showed that electrically coupled L14 neurons cannot pass Lucifer yellow, but can pass the smaller tetraalkylammonium and triethano- lamine ions (Bodmer et al., 1988). Interestingly, these investigators found that L14 neurons are better coupled in situ than in vitro, and are less sensitive to uncoupling agents, such as hydrogen ions, in the ganglion than in the dish. In cultured mouse spinal cord, pairs of astrocytes or pairs of oligodendrocytes are well coupled electrically and are dyc coupled with Lucifer yellow, but mixed pairs (astrocyte-oligodendrocyte) show weaker electrical coupling and a complete absence of detectable dye coupling (Ransom and Kettenmann, 1990). These authors suggest that the weaker gap junctions may be more important in the passage of metabolites or signaling molecules than in providing electrical continuity between cells. We are also pursuing this idea in leech embryos.

Electrical coupling between embryonic AP neurons. The values for the coupling coefficients between AP cells were low, but not out of line with some other electrical synapses (e.g., Ransom and Kettenmann, 1990). It is likely that several factors contributed to the limited degree of coupling, and to the finding that some of the cells were not detectably coupled. First, the two somatic recording sites were electrotonically distant from the sites of the electrical synapses within the connective nerve. Since current can leak out of the entire length of membrane between the recording sites, we do not know the voltages across the gap junctions themselves. Therefore, our measurements of the coupling coefficient, which considers only voltages produced at the recording sites, were almost certainly underestimates. The low input resistances measured in these embryonic cells (averaging $6 \mathrm{M} \Omega$ at E 11) support this idea. Second, damage from the electrode penetrations themselves inmediately depolarized the embryonic neurons (from an average of $-23 \mathrm{mV}$ to $-14 \mathrm{mV}$, at F.1 1), possibly causing either voltage-dependent uncoupling (reviewed in Bennett and Verselis, 1992) or functional uncoupling due to a drop in extrajunctional resistance. Finally, there may be few gap junctional channels at the sites of contact between axons, or the ones that are there may have a low conductance. The experiments described here do not allow us to distinguish among these possibilities.

What is the role of transient gap junctions in development? This question remains unanswered today even though transient junctions have been thought to play a developmentally significant role since Potter et al. (1966) discovered them in the early squid embryo. Since then, they have been observed in a variety of cell types in many phyla, from echinoderms, mollusks, and arthropods to fish, amphibians, and mammals (reviewed in Warner, 1991). At carly stages of development, subsets of cells are often electrically coupled, dye coupled, or both, and the extent of this coupling changes with age (e.g., Bossinger and Schierenberg, 1992). It is thought that these early gap junctions facilitate communication between specific groups of cells by allowing signaling molecules to pass between them (reviewed in Warner, 1991). However, no identified signaling molecule has yet been shown to cross these junctions, though one might expect there to be a wide range of candidate molecules or ions that can pass (reviewed in Bennett and Versclis, 1992), including second messengers such as calcium and inositol 1,4,5-trisphosphate (Saez et al., 1989).

Information about gap junctions later in development, after neuronal differentiation is well underway, is thus far confined to the documentation of their appearance and subsequent disappearance. Transient gap junctions appear at the chick neuromuscular junction in vitro (Fischbach, 1972) and, interesting- 
ly, form only when muscle cells are isolated at a particular developmental age and state of innervation (Bonner, 1989). Transient junctions are also formed between groups of neurons, as in the rat neocortex, where roles for gap junctions in synchronization of electrical activity (Peinado et al., 1993) and in the generation of columnar architecture (Yuste et al., 1992) have been postulated. In the Daphnia visual system, a pioneer axon (LoPresti et al., 1973) forms transient gap junctions with undifferentiated neurons in the optic lamina (LoPresti et al., 1974), and it is suggested that a signal might cross from the pioneer to a neuron, telling it when or how to differentiate (LoPresti et al., 1974). Similarly, transjunctional signaling may be important for developing grasshopper pioneer neurons that are dye coupled to "guidepost" neurons, before the latter extend their axons (Taghert et al., 1982). In this system, calcium ions can cross these junctions when the guidepost cell is damaged, and possibly during normal development as well (Bentley et al., 1991).

Since calcium seems to be involved in the control of neurite outgrowth in vitro (see Kater and Mills, 1991, for review), the pursuit of calcium as a potential cross-junctional signal may be fruitful. Indeed, studies in our laboratory show that calcium ions can cross from one E11 AP cell to the other, via the gap junctions reported here to be formed between the transient axons in the connective nerve (Wolszon et al., 1994). The possible function of calcium in the reinitiation of outgrowth of the AP axon is currently under investigation.

A new and interesting idea about gap junctions in development is that they could function as extracellular recognition molecules, perhaps aiding the apposition of like membranes carrying the calcium-dependent adhesion molecule A-CAM (N-cadherin). Meyer et al. (1992) found that anti-connexin Fab fragments blocked the assembly of adherens junctions in Novikoff cells expressing connexin-43 and A-CAM and, interestingly, that antibodies to A-CAM blocked gap junction formation. This reciprocal interaction suggests that these membrane-spanning proteins, as well as others, could be arranged in specific cells in a way that one particular combination imparts a unique identity to a neuron. Like cells would therefore adhere to others of their kind (an AP to an AP, e.g.) if they express the same combination of extracellular moieties at the same time, thus providing a basis for age-dependent contact inhibition. When antibodies to the invertebrate homologs of these moieties become available, this idea will be testable in the nervous system of the embryonic leech.

\section{References}

Audesirk G, Audesirk T, Bowsher P (1982) Variability and frequent failure of Lucifer yellow to pass between two electrically coupled neurons in Lymnaea stagnalis. J Neurobiol 13:369-375.

Bandtlow CE, Schmidt MF, Hassinger TD, Schwab ME, Kater SB (1993) Role of intracellular calcium in NI-35-evoked collapse of neuronal growth cones. Science 259:80-83.

Bennett MVL, Verselis VK (1992) Biophysics of gap junctions. Semin Cell Biol 3:29-47.

Bentley D, Guthrie PB, Kater SB (1991) Calcium ion distribution in nascent pioneer axons and coupled preaxonogenesis neurons in situ. J Neurosci 11:1300-1308

Bodmer R, Verselis V, Levitan IB, Spray DC (1988) Electrotonic synapses between Aplysia neurons in situ and in culture: aspects of regulation and measurements of permeability. $J$ Neurosci 8:16561670.

Bonner PH (1989) Correlation of development stage and gap junction formation between chick embryo neurons and cloned skeletal muscle myoblasts. Exp Cell Res 181:205-216.
Bossinger O, Schicrenberg E (1992) Cell-ccll communication in the embryo of Caenorhabditis elegans. Dev Biol 151:401-409.

Caroni P, Schwab ME (1988) Two membrane protein fractions from rat central myelin with inhibitory properties for neurite growth and fibroblast spreading. J Cell Biol 106:1281-1288.

Coggeshall RE (1974) Gap junctions between identified glial cells in the leech. J Neurobiol 5:463-467.

Fischbach GD (1972) Synapse formation between dissociated nerve and muscle cells in low density cell cultures. Del Biol 28:407-429.

Gao W-Q (1989) Axonal extension and retraction by developing neurons in the leech central nervous system. Ph.D. thesis, Columbia University.

Gao W-Q, Macagno ER (1987a) Extension and retraction of axonal projections by some developing neurons in the leech depends upon the existence of neighboring homologues. I. The HA cells. J Neurobiol 18:43-59.

Gao W-Q, Macagno ER (1987b) Extension and retraction of axonal projections by some developing neurons in the leech depends upon the existence of neighboring homologues. II. The AP and AE cells. J Neurobiol 18:295-313.

Haydon PG, McCobb DP, Kater SB (1984) Serotonin selectively inhibits growth cone motility and the connection of identified neurons. Science 226:561-564.

Kapfhammer JP, Raper JA (1987a) Collapse of growth cone structure on contact with specific neurites in culture. J Neurosci 7:201-212.

Kapfhammer JP, Raper JA (1987h) Interactions between growth cones and neurites growing from different neural tissues in culture. $J$ Neurosci 7:1595-1600.

Kater SB, Mills LR (1991) Regulation of growth cone behavior by calcium. J Neurosci 11:891-899.

Lankford DL, DeMello FG, Klein WL (1988) Dl-type dopamine receptors inhibil growth cone motility in cultured retina neurons: evidence that neurotransmitters act as morphogenic growth regulators in the developing central nervous system. Proc Natl Acad Sci USA $85: 2839-2843$.

Letourneau PC, Kater SB, Macagno ER (1992) The nerve growth cone. New York: Raven.

LoPresti V, Macagno ER, Levinthal C (1973) Structure and development of neuronal connections in isogenic organisms: cellular interactions in the development of the optic lamina of Daphnia. Proc Natl Acad Sci USA 70:433-437.

LoPresti V, Macagno ER, Levinthal C (1974) Structure and development of neuronal connections in isogenic organisms: transient gap junctions between growing optic axons and lamina neuroblasts. Proc Natl Acad Sci USA 71:1098-1 102.

Macagno ER (1980) Number and distribution of neurons in leech segmental ganglia. J Comp Neurol 190:283-302.

Macagno ER, Levinthal C, Sobel I (1979) Three-dimensional computer reconstruction of neurons and neuronal assemblies. Annu Rev Biophys Bioeng 8:323-351.

Maranto AR (1982) Neuronal mapping: a photooxidation reaction makes Lucifer yellow useful for electron microscopy. Science 217: 953-955.

McGlade-McCulloh E, Muller KJ (1989) Developing axons continue to grow at their tip after synapsing with their appropriate target. Neuron 2:1063-1068.

Meyer RA, Laird DW, Revel J-P, Johnson RG (1992) Inhibition of gap junction and adherens junction assembly by connexin and A-CAM antibodies. J Cell Biol 119:179-189.

Muller KJ, Scott SA (1981) Transmission at a "direct" electrical connexion mediated by an interneurone in the leech. J Physiol (Lond) 311:565-584.

Muller KJ, Nicholls JG, Stent GS (1981) Neurobiology of the leech. Cold Spring Harbor, NY: Cold Spring Harbor Laboratory.

Passani MB (1992) Developmental strategies in the establishment of central and peripheral projections by leech neurons. Ph.D. thesis, Columbia University.

Patterson P (1988) On the importance of being inhibited, or saying No to growth cones. Neuron 1:263-267.

Peinado A, Yuste R, Katz LC (1993) Extensive dye coupling between rat neocortical neurons during the period of circuit formation. Neuron 10:103-114.

Popov S, Brown A, Poo M-M (1993) Forward plasma membrane flow in growing nerve processes. Science 259:244-246.

Potter DD, Furshpan EJ, Lennox ES (1966) Connections between cells 
of the developing squid as revealed by electrophysiological methods. Proc Natl Acad Sci USA 55:328-335.

Pumplin DW, Muller KJ (1983) Distinctions between gap junctions and sites of intermediate filament attachment in the leech C.N.S. J Neurocytol 12:805-815.

Ransom BR, Kettenmann H (1990) Electrical coupling, without dye coupling, between mammalian astrocytes and oligodendrocytes in cell culture. Glia 3:258-266.

Raper JA, Kapfhammer JP (1990) The enrichment of a neuronal growth cone collapsing activity from cmbryonic chick brain. Neuron 2:21-29.

Ready DF, Nicholls J (1979) Identified neurones isolated from leech CNS make selective connections in culture. Nature 281:67-69.

Saez JC, Connor JA, Spray DC, Bennett MVL (1989) Hepatocyte gap junctions are permeable to the second messenger, inositol 1,4,5-trisphosphate, and to calcium ions. Proc Natl Acad Sci USA 86:27082712.

Schwab ME, Kapfhammer JP, Bandtlow CE (1993) Inhibitors of neurite growth. Annu Rev Neurosci 16:565-595.

Scott SA, Muller KJ (1980) Synapse regeneration and signals for directed axonal growth in the central nervous system of the leech. Dev Biol 80:345-363.
Stevens JK, Trogadis J (1984) Computer-assisted reconstruction from serial electron micrographs: a tool for the systematic study of neuronal form and function. In: Advances in cellular neurobiology, $\mathrm{Vol} 5, \mathrm{pp}$ 341-369. New York: Academic.

Taghert PH, Bastiani MJ, Ho RK, Goodman CS (1982) Guidance of pioneer growth cones: filopodial contacts and coupling revealed with an antibody to Lucifer yellow. Dev Biol 94:391-399.

Wadepuhl M (1989) Depression of excitatory motoneurones by a single neurone in the leech central nervous system. J Exp Biol 143:509_ 527.

Warner A (1992) Gap junctions in development-a perspective. Semin Cell Biol 3:81-91.

Wenning A (1987) Salt and water regulation in Macrobdella decorata (Hirudinea: Gnathobdelliformes) under osmotic stress. J Exp Biol 131:337-349.

Wolszon LR, Rehder V, Kafer SB, Macagno ER (1994) Calcium wavefronts that cross gap junctions may signal neuronal death during development. J Neurosci, in press.

Yuste R, Peinado A, Katz LC (1992) Neuronal domains in developing neocortex. Science 257:665-669. 